\title{
SEQUENTIAL WEAK CONVERGENCE IN THE BIDUAL OF A BANACH LATTICE
}

\author{
JOHN L. B. GAMLEN ${ }^{1}$
}

ABSTRACT. Let $X$ be a Banach lattice with separable dual. Then the bidual $X^{\prime \prime}$ is the closure of $X$ under sequential monotone weak convergence.

This paper proves a special case of a conjecture relating two types of weak sequential closure in the bidual $X^{\prime \prime}$ of a Banach lattice $X$. Specifically, if $Y$ is a sublattice of $X^{\prime \prime}$, write $Y^{\sigma}$ for the smallest subset of $X^{\prime \prime}$ which contains $Y$ and is closed under sequential monotone $\sigma\left(X^{\prime \prime}, X^{\prime}\right)$ convergence. It turns out that $Y^{\sigma}$ is a sublattice. We will study its relation with the space $\tilde{Y}$ generated by sequential $\sigma\left(X^{\prime \prime}, X^{0}\right)$ convergence.

Conjecture. The spaces $X^{\sigma}, \widetilde{X}$ coincide.

We prove a special case below (Theorem 1). Incidentally, note that, for a more general sublattice $Y$, the spaces $Y^{\sigma}, \widetilde{Y}$ may not coincide. An example is with $X=L^{1}[0,1]$. Let $K$ be the maximal ideal space of $L^{\infty}[0,1]$, and identify $X^{\prime \prime}$ with the regular measures on $K$. Take $Y$ to be the atomic measures, noting that $Y^{\sigma}=Y$, but $\tilde{Y}=X^{\prime \prime}$.

Theorem 1. If $X$ is a Banacb lattice with separable dual, and $Y$ is a sublattice of $X^{\prime \prime}$, then $Y^{\sigma}=\tilde{Y}$. In particular $X^{\sigma}=\tilde{X}=X^{\prime \prime}$.

Another special case of the conjecture is known already, and is a deep result of T. Ogasawara [6] stating (essentially) that if $X$ is a Banach lattice in which every monotone bounded sequence converges weakly, then $X$ is we akly complete. This may be rephrased: "if $X^{\sigma}=X$, then $\widetilde{X}=X^{\prime \prime}$ ".

The space $C[0,1]$ is a nice nontrivial example of a Banach lattice in which the conjecture holds. In this case both $X^{\sigma}, \widetilde{X}$ may be identified with the space of bounded Borel-measurable functions on $[0,1]$. It is easy to see that a sequence of such functions converges for $\sigma\left(C^{\prime \prime}, C^{\prime}\right)$ if and only if it is uniformly bounded and pointwise convergent.

If $Y$ is a linear subspace of $X^{\prime \prime}$, write $Y^{\Sigma}$ for the smallest subspace of $X^{\prime \prime}$ containing $Y$ and closed under weak unconditional series convergence. It is clear that, in general,

Received by the editors March 26, 1973.

AMS (MOS) subject classifications (1970). Primary 46A40, 46B99.

Key words and phrases. Weak sequential convergence.

1 The author is partially supported by N.R.C. Grant A7552 and NSF GP-30671. 


$$
X^{\sigma} \subseteq X^{\Sigma} \subseteq \tilde{X} \subseteq X^{\prime \prime}
$$

Note here that monotone weak limits may be expressed as limits of positive term series which converge unconditionally (weakly) because members of $X^{\prime}$ are differences of positive members. Thus Theorem 1 implies

Corollary 2. For a Banach lattice with separable dual, equality holds in (*).

This result can be used to give another proof of the following result of T. Ogasawara [6]:

Corollary 3. If $X$ is a Banach lattice with separable bidual then $X$ is reflexive.

Proof of Corollary 3. Since $X^{\prime \prime}$ is separable, it is clear that $X$ cannot have a subspace isomorphic to $c_{0}$, and thus by a theorem of Bessaga and Pelczynski [1], $X^{\Sigma}$ must coincide with $X$. Corollary 2 now shows $X^{\prime \prime}=X$.

One can also argue above using Ogasawara's first-mentioned result, instead of Corollary 2. (Since $X^{\boldsymbol{\Sigma}}=X$, bounded monotone sequences converge weakly so $X$ is weakly complete, but $X^{\prime}$ is separable, proving reflexivity.)

It is well known that Corollary 3 does not extend to general Banach spaces, as the famous counterexample of R. C. James [3] shows.

For expositions of the elementary Banach lattice theory we will use, the reader is referred to [2] or [9]. For further results in the spirit of Ogasawara's theorems, but in a more general setting, the $r \epsilon$ ader is referred to the recent paper of L. Tzafriri [8].

The proof of Theorem l. For simplicity we deal only with the case $Y=X$. Once we know that $X^{\sigma}=X^{N}$, treatment of the general sublattice $Y$ is just an imitation.

Lemma 4. If $X$ is a Banach lattice, then $X^{\sigma}$ is a sublattice of $X^{\prime \prime}$.

Proof. The idea is the same as in the analogous result for Boolean algebras (the "monotone class" theorem).

Let $X_{1}=\left\{x\right.$ in $\left.X^{\prime \prime}: x \vee 0 \in X^{\sigma}\right\}$. A routine argument shows that $X_{1}$ is closed under monotone sequential $\sigma\left(X^{\prime \prime}, X^{\theta}\right)$ convergence. This means $X_{1}$ contains $X^{\sigma}$, which shows $X^{\sigma}$ is a sublattice.

We introduce a new topology $\mathcal{T}$ on $X^{\prime \prime}$, defined by the seminorms $p_{\phi}, \phi \in X^{\prime}$ where $p_{\phi}(x)=\langle|x|,|\phi|\rangle$ for $x \in X^{\prime \prime}$. Incidentally, this is the "locally solid" topology generated by $\sigma\left(X^{\prime \prime}, X^{\prime}\right)$ in the sense of $\mathrm{I}$. Namioka [5].

Lemma 5. (a) $X$ is dense in $\left(X^{\sigma}, \mathcal{T}\right)$.

(b) The weak topology of $\left(X^{\sigma}, \mathfrak{T}\right)$ is $\sigma\left(X^{\sigma}, X^{\prime}\right)$.

Proof. From the definition, monotone convergence for $\mathcal{T}$ and $\sigma\left(X^{\prime \prime}, X^{*}\right)$ coincide. This proves (a).

Clearly the members of $X^{\prime}$ are $\mathcal{S}$-continuous functionals. For the con- 
verse note that each $\mathcal{T}$-continuous linear functional $\psi$ on $X$ has a restriction to $X$ which is a member $\phi$ of $X^{\circ}$. By part (a), $\phi$ and $\psi$ coincide on $X^{\sigma}$.

The proof of Theorem 1 goes as follows. Let $\left\{\phi_{n}\right\}$ be a sequence of positive norm-one members of $X^{\prime}$ with dense span. Construct an increasing norm $\rho$ on $X^{\sigma}$ as follows.

Define

$$
\rho(x)=\sum_{1}^{\infty} 2^{-n}\left\langle|x|, \phi_{n}\right\rangle \text { for } x \in X^{\prime \prime} .
$$

It is clear that $\rho$ gives the same topology as $\mathcal{T}$ on bounded subsets of $X^{\prime \prime}$. Write $L$ for the completion of $X$ under the norm $\rho$. Not only is $L$ a Banach lattice, but by Kakutani's theorem [4] $L$ is isomorphic to some $L^{1}(\mu)$, and is thus weakly complete. We make two claims:

(1) the weak topology of $L$ coincides on bounded subsets of $X^{\sigma}$ with $\sigma\left(X^{\sigma}, X^{\prime}\right)$

(2) the unit ball $B$ in $X^{\sigma}$ is $\rho$-complete, and hence by (1) sequentially complete for $\sigma\left(X^{\sigma}, X^{\prime}\right)$.

Obviously (1), (2) finish the proof of Theorem 1.

Proof of Claim (1). Since the topology of $\rho$ is weaker than $\mathcal{J}$ (on $X^{\prime \prime}$ ), the weak topology of $L$ is weaker than the weak topology of $\left(X^{\sigma}, \mathcal{T}\right)$, which by Lemma 5 is $\sigma\left(X^{\sigma}, X^{0}\right)$ on $X^{\sigma}$. For the converse, note that the functionals $\phi_{n}$ are $\rho$-continuous, and on bounded sets they determine the topology $\sigma\left(X^{\sigma}, X^{\prime}\right)$.

Proof of Claim (2). Let $\left\{x_{n}\right\}$ be a $\rho$-Cauchy sequence in $B$, say $\left\{x_{n}\right\}$ converges in $L$ to $x_{\infty}$ Since $x_{n}^{+}$converges in $L$ to $x^{+}$, we may assume that $x_{n}, x$, are positive, and each $x_{n}$ is in $B$. Write $y_{k}=\bigvee_{n=k}^{\infty}{ }_{n}$, which exists as a monotone $\sigma\left(X^{\prime \prime}, X^{\prime}\right)$ limit, and thus lies in $X^{\sigma}$. Let us assume that $\rho\left(x_{n}-x\right)<2^{-n}$, all $n$. We claim that $\rho\left(y_{k}-x_{k}\right)<2^{2-k}$, all $k$. To prove this, write $y_{k}-x_{k}$ as $\bigwedge_{n=k}^{\infty}\left(x_{n}-x_{k}\right)$, to obtain:

$$
\rho\left(y_{k}-x_{k}\right) \leq \rho\left(\sum_{n=k}^{\infty}\left|x_{n}-x_{k}\right|\right)<2^{2-k}, \text { as required. }
$$

(The series on the right converges for $\rho$ and for $\sigma\left(X^{\prime \prime}, X^{\prime}\right)$ to an element of $X^{\sigma}$. The fact that $\rho$ is an increasing norm then yields the above inequality.)

Claim (2) now follows from the observation that $\left\{y_{k}\right\}$ is a monotone increasing sequence in $B$, so must converge for $\rho$ and for $\sigma\left(X^{\prime \prime}, X^{\prime}\right)$ to a member of $B$ which the above reasoning shows is $x$.

Remarks on the proof of Theorem 1. It might be thought that the Theorem could be proved directly by some variant of the above argument involving $\left\{y_{k}\right\}$. The trouble is that if $x_{k}$ converges to $x$ for $\sigma\left(X^{\prime \prime}, X^{\prime}\right)$, then $x_{k}^{+}$ need not converge to $x^{+}$. (For example, in $L^{2}[0, \pi], x_{k}(t)=\sin k t$.) The point of our proof is that $\rho$-convergence does not suffer from this defect. It might also occur to the reader that a theorem of H. Schaefer (cf. A. 
Peressini [7, p. 146]) concerning metrizable locally convex lattices could be modified to deal with our situation. This fails because the weak topology $\sigma\left(X^{\prime \prime}, X^{\prime}\right)$ is not metrizable, even though the bounded sets are metrizable.

Our results raise the question of which sequent ially monotone complete topological vector lattices are already sequentially complete? The facts we have used about $X^{\sigma}, \sigma\left(X^{\sigma}, X^{\prime}\right)$ in proving its seque ntial completeness are:

(a) $\sigma\left(X^{\sigma}, X^{\prime}\right)$ is the weak topology of a locally solid space;

(b) the bounded sets are metrizable (for the weak topology, in this case $\left.\sigma\left(X, X^{*}\right)\right)$.

Unfortunately (a) may fail even for the topology $\sigma\left(X^{\prime}, X\right)$ of the dual $X^{\prime}$ of a Banach lattice $X$. (Take $X=C[0,1]$. The locally solid topology generated by $\sigma\left(X^{0}, X\right)$ is the norm topology, whose weak topology is not $\sigma\left(X^{\prime}, X\right)$.)

\section{REFERENCES}

1. C. Bessaga and A. Pelczynski, On bases and unconditional convergence of a series in Banach spaces, Studia. Math. 17 (1958), 151-164. MR 22 \#5872.

2. Garrett Birkhoff, Lattice theory, 3rd ed., Amer. Math. Soc. Colloq. Publ., vol. 25, Amer. Math. Soc., Providence, R. I., 1967. MR 37 \#2638.

3. R. C. James, A non-reflexive Banach space isometric with its second conjugate space', Proc. Nat. Acad. Sci. U.S.A. 37 (1951), 174-177. MR 13, 356.

4. S. Kakutani, Concrete representation of abstract $(L)$-spaces and the mean ergodic theorem, Ann. Math. (2) 42 (1941), 523-537. MR 2. 318.

5. I. Namioka, Partially ordered linear topological spaces, Mem. Amer. Math. Soc. No. 24 ( 1957). MR 20 \#1193.

6. T. Ogasawara, Theory of vector lattices. I, J. Sci. Hiro shima Univ. Ser. A 12 (1942), 37-100. MR 10, 545 .

7. A. Peressini, Ordered topological vector spaces, Harper and Row, New York, 1967. MR $37 \# 3315$.

8. L. Tzafriri, Reflexivity in Banach lattices and their subspaces, J. Functional Analysis 10 (1972), 1-18.

9. A. C. Zaanen, and W. A. J. Luxemburg, Riesz spaces, North-Holl and, London, 1971.

DEPARTMENT OF MATHEMATICS, YALE UNIVERSITY, NEW HAVEN, CONNECTICUT 06520

DEPARTMENT OF MATHEMATICS, UNIVERSITY OF ALBERTA, EDMONTON, AL• BERTA, CANADA 